\title{
Can Acute Stress Cause Esophageal Hypersensitivity in Healthy Individuals?
}

\author{
Yu Kyung Cho \\ Department of Internal Medicine, The Catholic University of Korea, College of Medicine, Seoul, Korea
}

\author{
Article: Intravenous corticotropin releasing hormone administration increases esophageal electrical sensitivity in healthy \\ individuals \\ Yamasaki T, Tomita T, Takimoto M, et al \\ (J Neurogastroenterol Motil 2017;23:526-532)
}

Stress exacerbates heartburn symptoms in gastroesophageal reflux disease (GERD) patients and also affects visceral sensitivity. ${ }^{1}$ The mechanism of visceral hypersensitivity in functional gastrointestinal disorders and functional heartburn remains unclear. However, the involvement of stress in relation to esophageal sensitivity has been reported. ${ }^{1,2}$ Fass et $\mathrm{al}^{3}$ demonstrated that GERD patients who were exposed to acute auditory stress experienced increased levels of heartburn induced by intraesophageal acid perfusion, and the stress from disturbed sleep has also been shown to cause similar esophageal hypersensitivity.

In the revised Rome IV criteria, functional esophageal disorders include functional chest pain, functional heartburn, reflux hypersensitivity, globus, and functional dysphagia. ${ }^{4}$ The Rome IV adopts a more rigorous definition of GERD. GERD patients are subgrouped as erosive esophagitis, non-erosive reflux disease (NERD), reflux hypersensitivity, and functional heartburn. ${ }^{4}$ The pathophysiology of erosive esophagitis and NERD is the result of pathologic acid exposure in contrast to the functional disorders that are related to esophageal hypersensitivity. Esophageal hypersensitivity is one of the important mechanisms in GERD patients who are refractory to acid-suppressive therapy. There is an association between distal esophageal acid exposure and esophageal hypersensitivity among the reflux-related disorders.

Esophageal pain can be induced by mechanical distension, chemical stimuli, and others. A high percentage of patients with functional chest pain reported pain after balloon distension of the esophagus. ${ }^{6}$ Enhanced chemosensitivity in functional heartburn patients has also been reported. ${ }^{7}$ Factors that influence visceral hypersensitivity in the esophagus, include stress, mucosal integrity, and microinflammation in the esophagus, though the mechanisms by which visceral hypersensitivity of the esophagus occurs are incompletely understood. ${ }^{1}$

Corticotropin-releasing hormone (CRH) plays a key role in the acute regulation of stress- and anxiety-related behaviors and in the regulation of endocrine responses during chronic stress via activation of the hypothalamic-pituitary-adrenal axis. Peripheral CRH is a key mediator of the gut stress response, and has been shown to influence visceral hypersensitivity in humans. ${ }^{8}$ Stress affects esophageal motility. A previous study demonstrated that intravenous $\mathrm{CRH}$ administration enhanced esophageal sensitivity to mechani-

Received: September 23, 2017 Revised: None Accepted: September 26, 2017

(c) This is an Open Access article distributed under the terms of the Creative Commons Attribution Non-Commercial License (http://creativecommons. org/licenses/by-nc/4.0) which permits unrestricted non-commercial use, distribution, and reproduction in any medium, provided the original work is properly cited.

${ }^{*}$ Correspondence: Yu Kyung Cho, MD

Department of Internal Medicine, The Catholic University of Korea, College of Medicine, Seoul, Korea Tel: +82-2-2258-6017, Fax: +82-2-2258-2064, E-mail: ykcho@catholic.ac.kr 
cal distension, increased esophageal contractility, and decreased LES relaxation, therefore improving esophageal bolus clearance. ${ }^{8}$ The changes after $\mathrm{CRH}$ administration in esophageal contractile properties may be associated with the increased sensitivity to balloon distention after $\mathrm{CRH}$.

Stress is able to alter esophageal sensitivity. In this issue of the Journal of Neurogastroenterology and Motility, Yamasaki et al ${ }^{9}$ demonstrated CRH administration increased esophageal electrical sensitivity in normal subjects, emphasizing the important role of stress in esophageal sensitivity. The results of this study were meaningful in studying the mechanisms of esophageal functional pain in refractory GERD or functional heartburn.

There is increasing evidence that esophageal hypersensitivity in patients with proton proton pump inhibitor (PPI)-refractory GERD symptoms is of a multisensory nature. Many solutions are suggested as the next therapeutic or diagnostic option in PPIrefractory NERD patients. Some of these patients proved to have functional heartburn and reflux hypersensitivity. Esophageal hypersensitivity will be a new therapeutic target in addition to PPIs for those patients.

\section{Financial support: None.}

\section{Conflicts of interest: None.}

\section{References}

1. Kondo T, Miwa $\mathrm{H}$. The role of esophageal hypersensitivity in functional heartburn. J Clin Gastroenterol 2017;51:571-578.

2. Farmer AD, Ruffle JK, Aziz Q. The role of esophageal hypersensitivity in functional esophageal disorders. J Clin Gastroenterol 2017;51:91-99.

3. Fass R, Naliboff BD, Fass SS, et al. The effect of auditory stress on perception of intraesophageal acid in patients with gastroesophageal reflux disease. Gastroenterology 2008;134:696-705.

4. Aziz Q, Fass R, Gyawali CP, Miwa H, Pandolfino JE, Zerbib F. Functional esophageal disorders. Gastroenterology Published Online First: 15 Feb 2016. doi: 10.1053/j.gastro.2016.02.012.

5. Nasr I, Attaluri A, Hashmi S, Gregersen H, Rao SS. Investigation of esophageal sensation and biomechanical properties in functional chest pain. Neurogastroenterol Motil 2010;22:520-526, e116.

6. Farmer AD, Coen SJ, Kano M, et al. Psychophysiological responses to visceral and somatic pain in functional chest pain identify clinically relevant pain clusters. Neurogastroenterol Motil 2014;26:139-148.

7. Tamura Y, Funaki Y, Izawa S, et al. Pathophysiology of functional heartburn based on Rome III criteria in Japanese patients. World J Gastroenterol 2015;21:5009-5016.

8. Broers C, Melchior C, Van Oudenhove L, et al. The effect of intravenous corticotropin-releasing hormone administration on esophageal sensitivity and motility in health. Am J Physiol Gastrointest Liver Physiol 2017;312:G526-G534.

9. Yamasaki T, Tomita T, Takimoto M, et al. Intravenous corticotropin releasing hormone administration increases esophageal electrical sensitivity in healthy individuals. J Neurogastroenterol Motil 2017;23:526-532. 